\title{
Microring Resonator Refractive Index Sensor with Integrated Spectrometer
}

\author{
Alfonso Ruocco, José Juan Colás and Wim Bogaerts \\ Photonics Research Group, Ghent University - imec,Department of Information Technology \\ Center of Nano- and Biophotonics (NB-photonics), Ghent, Belgium \\ Email: Alfonso.Ruocco@intec.UGent.be
}

\begin{abstract}
We present a SOI ring based sensor read-out system. The novelty of the architecture lies in the capability to sense the shifts of multiple peaks simultaneously with an integrated AWG spectrometer.
\end{abstract}

\section{INTRODUCTION}

SOI photonic sensors proved themselves to be very effective for a variety of applications. The high refractive index contrast keeps the size very compact and the silicon material system allows for mass production. Most photonic sensors are based on the refractive index change. Despite the reduced dimensions and potentially low cost of the sensor itself, the interrogating structure remains quite expensive, and often bulky. In this paper we introduce a microring sensor and an integrated AWG (Arrayed Waveguide Grating) based spectrometer. The proposed architecture can use a broadband source such as an SLED instead of a bulky and costly tunable CW laser. The FSR (Free Spectral Range) of the microring and the channel spacing of the AWG are defined according to a Vernier-like scaling. This configuration amplifies the shift of the ring peak with a factor of 8.85 .

\section{The Microring RESONATOR SENSOR}

The response of a MRR (Micro-Ring Resonator) sensor is a comb-like transmission spectrum with peaks at the resonance wavelengths and spaced proportionally to the optical group roundtrip delay of the device [1]. Anything that affects the effective index of the ring waveguide will effect a wavelength shift of the resonances. The change in effective index can be induced by a change in the overcladding (like a fluid) or binding of biological molecules. For this experiment, we simple add a heater on top of the sensor ring, as this allows us to reproducibly test the concept of our device. We use thin silicon rib waveguides. The high thermo-optic coefficient of silicon will affect the waveguide's effective index and shift the resonance wavelengths. Titanium heaters are processed on the waveguide constituting the microring to electrically control the temperature.

\section{THE AWG INTERROGATOR}

The interrogator of the microring sensor consists of an AWG. This component is often used for waveguide division multiplexing but recently also adopted more other applications such as sensing and wavelength analysis [2]. It consists of two FPRs (Free Propagation Region) and an array of waveguides.

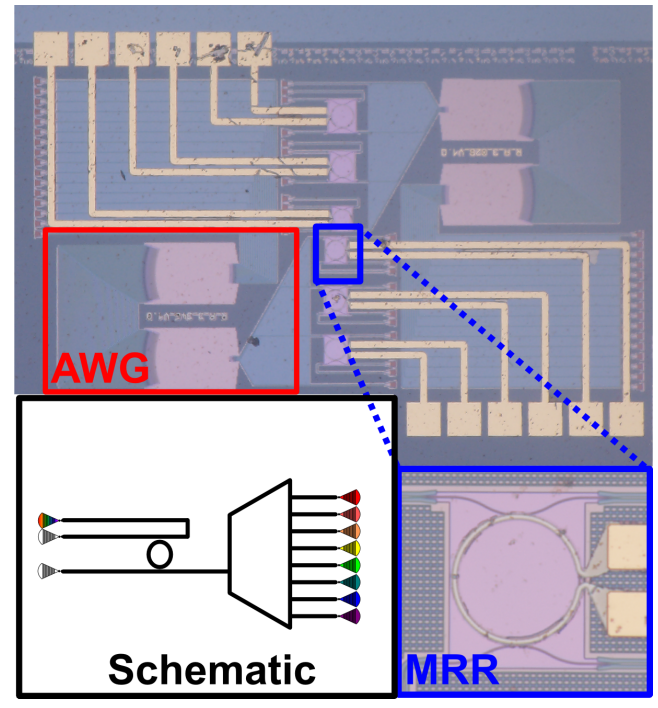

Fig. 1: Microscope image and schematic of the PIC

The physical path increment between the waveguides is constant. Each output channel exhibits a Gaussian-like wavelength transmission. The FSR of the AWG is the wavelength separation between $N^{t h}$ and the $N^{t h}+1$ diffraction order in the same physical output channel. Our AWG has an FSR of 48 $\mathrm{nm}$ and $\mathrm{m}=16$ channels separated with a $\Delta \lambda_{c h}=3.0 \mathrm{~nm}$.

The relation in between the channel spacing $\Delta \lambda_{c h}$ of the AWG and the FSR of the ring are designed according to

$$
F S R_{\text {ring }}=\Delta \lambda_{c h} \frac{m}{m-1}
$$

The factor $m / m-1$ results in a Vernier effect in comb of resonance peaks of the ring and the output channels of the AWG [2]. The output power of each AWG channel is represented by

$$
T=\sum_{m=0}^{M}\left(\sum_{n=0}^{N} L_{r i n g}\left(\lambda-\lambda_{n}\right)\right) G_{A W G}\left(\lambda-\lambda_{m}\right)
$$

where $L$ is the Lorentzian-like response of the ring and $G$ the Gaussian-like response of the AWG channel. When the FSR of the ring is the same as the AWG channel spacing, the output collected will be the same for all channels. But when we introduce the Vernier factor [3], we will obtain a well defined 


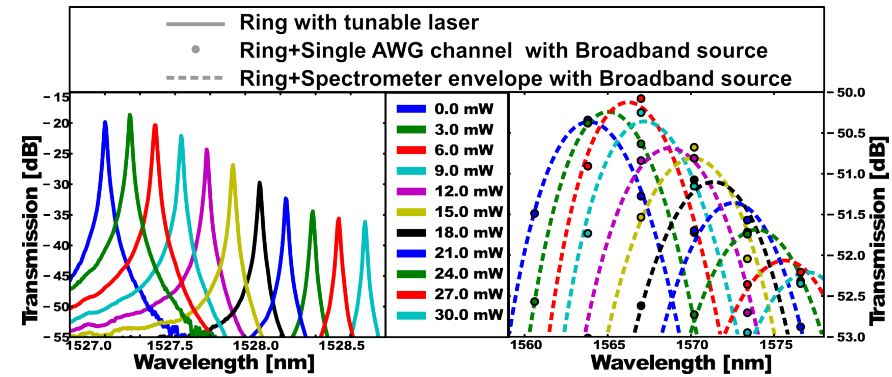

Fig. 2: (a)Ring sensor and (b)PIC envelope $\Delta \lambda$ shift

envelope over the channels of the AWG. We use a Vernier factor of $m / m-1$, but it is also possible to use $m / m-2, \ldots$ These will give envelopes with multiple maxima. Fig. 2 right plot represents the envelope shift induced by thermo-optic effect and the relative fitting, while the left plot represents the shift of one single peak if the same ring sensor is used standalone. The relative spectral position of the AWG channels and the comb of the ring peaks determines the maximum of the envelope. But because of the vernier effect, the shift of the envelope is theoretically limited to $m-1$ times larger than the shift of the ring comb.

\section{Characterization AND RESUlts}

If the ring acts as a sensor, the sensitivity of the readout is enhanced. We can induce a wavelength shift in the comb of the ring by driving a power through the Titanium heater. We can read out the shift of the ring itself directly by monitoring the spectrum of the pass port using a grating coupler. the drop port of the ring 'sensor' is connected to the input of the AWG. The outputs of the AWG are characterized using a semi-automatic electro-optic measurement setup: both optical and electrical in-out are computer managed. Since the peak shift is linearly related with the thermal power, the chosen driving scheme used is step-by-step power sweep. The used source is a SLED centered an $1550.0 \mathrm{~nm}$. The operating range of the circuit is limited to a single FSR of the AWG, so we used a tunable optical bandpass filter is used to limit the band of the source and avoid aliasing effects of the outputs. We measured the AWG outputs sequentially, performing a controlled sweep of the thermal power on the ring. The final obtained correlation is between the electrical power biasing the heater and the optical power collected in each of the $N$ AWG outputs. Fig.2 represents the wavelength shift of a single peak of a ring on the left, while on the right the fitted envelope of the full AWG circuit is considered. The shift of the envelope is 8.85 times larger than that of the ring, this corresponds to the $G$ in the eq.3. The gain factor in smaller then what expected due to processing related specs variations of ring and AWG and to the stringent requirements of the design. The envelope plot is obtained normalizing the spectrum to the wavelengths of the AWG channels obtained during the characterization phase, this is required since we are using a broadband source and no wavelength information are available. This improvement can

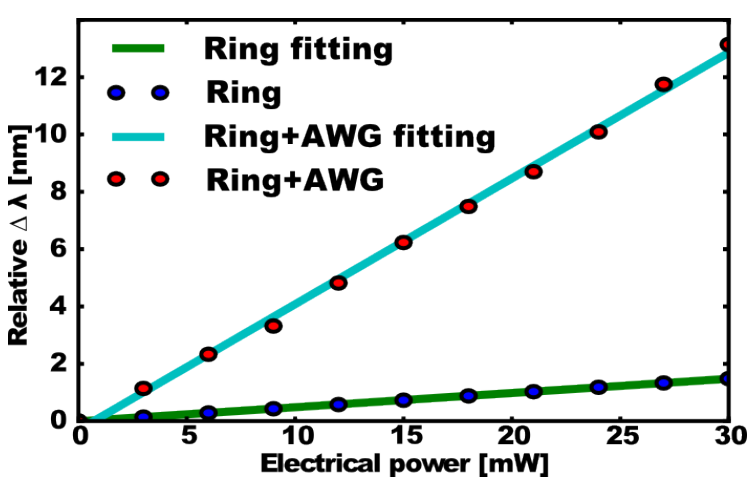

Fig. 3: Relative $\Delta \lambda$ versus input electrical power

be translated in a gain of the limit-of-detection (LOD), or the smallest wavelength shift that can be measured:

$$
L O D=\frac{\Delta \lambda_{\min }}{G \frac{d \lambda}{d R I U}}
$$

The fig. 3 shows the relation between refractive index change through thermo-optic coefficient and the wavelength shift of the single ring and of the full circuit, showing again the strong improvement. An additional advantage is that the readout circuit is compatible with broadband sources, which can dramatically reduce the cost of the entire sensor system.

However, further investigation is ongoing about the behavior of this device in the presence of noise, and how more advanced calibration methods can be used to mitigate the imperfections in the ring and AWG subcircuits. Also, this device was demonstrated on a purely passive platform, but a significant improvement is expected with the use of integrated detectors.

\section{CONCLusions}

We designed, simulated and characterized a novel interrogation circuit for comb-like sensor devices. The proof-of-concept has been tested with a ring used as a $n_{e f f}$ sensor. The results show a strong improvements of sensitivity and LOD of the system. Further investigation and optimization are required to validate our approach.

\section{ACKNOWLEDGEMENT}

The simulation and design have been carried out with Luceda Software. The silicon PIC was manufactured through the IMEC MPW fabrication service.

\section{REFERENCES}

[1] W. Bogaerts, P. De Heyn, T. Van Vaerenbergh et al., "Silicon microring resonators," Laser \& Photonics Reviews, vol. 6, no. 1, pp. 47-73, 2012. [Online]. Available: http://dx.doi.org/10.1002/lpor.201100017

[2] A. Ruocco, D. Van Thourhout, and W. Bogaerts, "Silicon photonic spectrometer for accurate peak detection using the vernier effect and timedomain multiplexing," Lightwave Technology, Journal of, vol. 32, no. 19, pp. 3351-3357, Oct 2014.

[3] T. Claes, W. Bogaerts, and P. Bienstman, "Experimental characterization of a silicon photonic biosensor consisting of two cascaded ring resonators based on the vernier-effect and introduction of a curve fitting method for an improved detection limit," Opt. Express, vol. 18, no. 22, pp. 22747-22 761, Oct 2010. [Online]. Available: http://www.opticsexpress.org/abstract.cfm?URI=oe-18-22-22747 